\title{
RELAÇÃO ENTRE ATRIBUTOS DE SOLOS E OXIDAÇÃO DE ENXOFRE ELEMENTAR EM QUARENTA E DUAS AMOSTRAS DE SOLOS DO BRASIL ${ }^{(1)}$
}

\author{
Nelson Horowitz ${ }^{(2)}$ \& Egon José Meurer ${ }^{(3)}$
}

\begin{abstract}
RESUMO
O uso do S-elementar como fertilizante pode reduzir custos de produção. É necessária, porém, a sua oxidação a S-sulfato, forma disponível à planta. Poucas informações são encontradas na literatura sobre a capacidade de solos brasileiros em oxidar S-elementar. Este trabalho foi realizado com os objetivos de determinar a taxa de oxidação de S-elementar em amostras de 42 solos coletadas em vários Estados do Brasil e verificar como atributos de solos podem afetar a oxidação. $O$ estudo foi realizado com amostras coletadas no horizonte $A$, na camada de 0 a $20 \mathrm{~cm}$, que foram incubadas com S-elementar na dose de $10 \mathrm{~g} \mathrm{~kg}^{-1} \mathrm{de} \mathrm{S}^{0}$ no solo por 90 dias em estufa na temperatura de $27 \pm 1^{\circ} \mathrm{C}$, em frascos de vidro com capacidade para $150 \mathrm{~mL}$. As amostras dos 42 solos apresentaram taxas de oxidação do Selementar a S-sulfato que variaram de 1,95 a $21,89 \mu \mathrm{g} \mathrm{cm}^{-2} \mathrm{dia}^{-1} \mathrm{de}^{0}$. A taxa de oxidação do S-elementar se correlacionou positivamente com o teor de matéria orgânica (MO) e negativamente com os teores de Al trocável e o teor inicial de S dos solos. $\mathrm{O}$ pH e os teores de argila, $\mathrm{P}, \mathrm{K}$ disponíveis, Ca e Mg trocáveis não afetaram a taxa de oxidação do S-elementar dos solos.
\end{abstract}

Termos de indexação: fertilizante, matéria orgânica, nutrientes, pH, taxas de oxidação, S-sulfato.

${ }^{(1)}$ Parte da Tese de Doutorado do primeiro autor submetida ao Programa de Pós-Graduação em Ciência do Solo da UFRGS. Recebido para publicação em dezembro de 2006 e aprovado em fevereiro de 2007.

(2) Engenheiro-Agrônomo, Doutor, Roullier Brasil. Av. Carlos Gomes 1340, CEP 90480-004 Porto Alegre (RS). E-mail: nelsontz@terra.com.br

(3) Professor do Departamento de Solos da Universidade Federal do Rio Grande do Sul - UFRGS. Caixa Postal 15100, CEP 91501970 Porto Alegre (RS). Bolsista do CNPq. E-mail: egon.meurer@ufrgs.br 


\title{
SUMMARY: RELATIONSHIP BETWEEN SOIL ATTRIBUTES AND ELEMENTAL SULFUR OXIDATION IN 42 SOIL SAMPLES FROM BRAZIL
}

\begin{abstract}
The use of elemental $S$ as fertilizer can reduce costs in agriculture, but elemental-S must first be oxidized to sulfate-S to become plant-available. Little information is found in the literature about the capacity of Brazilian soils to oxidize elemental-S. This study was carried out with the objective of determining the oxidation rates of 42 soils samples from different Brazilian states to verify how soil attributes affect oxidation. Samples were collected in the A horizon (0-20 cm layer) and incubated in $150 \mathrm{~mL}$ glass flasks with $10 \mathrm{~g} \mathrm{~kg}^{-1}$ of $S^{0}$ for 90 days, at $27 \pm 1{ }^{\circ} \mathrm{C}$. The samples of the 42 soils oxidized the elemental$S$ to sulfate-S at rates between 1.95 and $21.89 \mu \mathrm{g} \mathrm{cm}^{-2}$ day ${ }^{-1}$ of $S^{0}$. The oxidation rate was positively related to soil organic matter content and inversely related to exchangeable aluminum and initial sulfur content of the soils. Soil $\mathrm{pH}$ and soil concentration of phosphorus, potassium, calcium, and magnesium had no effect on the oxidation rate of elemental-S.
\end{abstract}

Index terms: fertilizer, organic matter, nutrients, $p H$, oxidation rates, sulfate-S.

\section{INTRODUÇÃO}

Deficiências de S ocorrem em diversas regiões do Brasil, notadamente nas áreas dos Cerrados. As fontes mais utilizadas para suprir o $\mathrm{S}$ às plantas são o superfosfato simples, que contém $12 \%$ de S-sulfato, e o sulfato de amônio, com $24 \%$ de S-sulfato. Ambos são utilizados isoladamente ou como componentes de fertilizantes comerciais. A utilização de S-elementar, que contém mais de $90 \%$ de $\mathrm{S}$, incorporado a fertilizantes comerciais é uma alternativa que pode aumentar a concentração do nutriente nas formulações e reduzir os custos de produção, de transporte e de aplicação do fertilizante.

Em diversos países, fertilizantes que contêm Selementar vêm sendo usados de forma crescente (Edmeades et al., 1994; Zhao et al., 1996). Fertilizantes com S-elementar são comercializados em países como Canadá, Austrália e Nova Zelândia. No Brasil, a sua utilização ainda é incipiente, embora algumas empresas comercializem o produto isoladamente ou em misturas fareladas. Contudo, após aplicado ao solo, é necessário que ele seja oxidado a S-sulfato, forma disponível à planta.

A taxa de oxidação do S-elementar é afetada por atributos químicos e físicos do solo, como aeração, pH, textura, teor de MO e teor de nutrientes (Germida \& Janzen, 1993), e pela biomassa microbiana (Germida \& Janzen, 1993). Vários trabalhos mostraram efeito estimulante da MO e do $\mathrm{P}$ na taxa de oxidação (Wainwright et al., 1986; Janzen \& Bettany, 1987a,b; Lawrence \& Germida, 1988; Cifuentes \& Lindemann, 1993; Cowell \& Schoenau, 1995; Lefroy et al., 1997; Sholeh et al., 1997). A disponibilidade de outros nutrientes, como o $\mathrm{N}$ e o $\mathrm{K}$, tem mostrado tanto efeito estimulante como supressivo sobre a oxidação; no caso da supressão, o efeito ocorre principalmente em altas concentrações, pois o excesso de sais inibe a oxidação (Keller, 1969). Outros trabalhos sugerem que a deficiência de nutrientes é raramente um fator que determina os valores das taxas de oxidação do Selementar (Watkinson, 1989). Diferentes espécies de plantas podem, também, afetar a taxa de oxidação do S-elementar, devido à variação das características da rizosfera entre espécies vegetais (Grayston \& Germida, 1990).

A ausência de uma relação bem definida entre a oxidação do S-elementar e as várias propriedades físicas e químicas do solo não implica, necessariamente, que esses efeitos não sejam importantes. É provável que a oxidação seja governada pela interação desses fatores e não apenas por um fator isolado, exceto em casos extremos (Germida \& Janzen, 1993).

Os objetivos deste trabalho foram: determinar a taxa de oxidação de S-elementar e verificar se o $\mathrm{pH}$ e

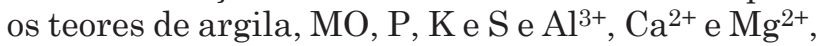
S e K do solo afetam a taxa de oxidação do S-elementar em amostras de 42 solos coletadas em vários Estados do Brasil.

\section{MATERIAL E MÉTODOS}

O trabalho foi realizado com 42 amostras de solos coletadas no Rio Grande do Sul (20), no Paraná (8), em São Paulo (6), no Piauí (3) e no Distrito Federal (5). As amostras foram coletadas no horizonte $\mathrm{A}$, na camada de 0 a $20 \mathrm{~cm}$. Após a coleta, foram postas a 
secar à sombra e, posteriormente, tamisadas em peneiras com malhas de $2,0 \mathrm{~mm}$ de abertura. No quadro 1 encontram-se alguns atributos das amostras dos solos, determinados conforme método descrito por Tedesco et al. (1995). O S-elementar utilizado foi previamente peneirado com etanol, que é um dispersante de S-elementar. Esse procedimento assegura a remoção de partículas finas aderidas às partículas do tamanho desejado (Janzen \& Bettany, 1987a). Com esse procedimento, o tamanho das partículas do S-elementar ficou entre 0,074 e 0,15 mm. Depois dessa tamisação em meio líquido, o S-elementar foi seco em estufa a $65^{\circ} \mathrm{C}$ e, a seguir, misturado na dose de $10 \mathrm{~g} \mathrm{~kg}^{-1}$ de $\mathrm{S}^{0}$ com as amostras dos solos secos que foram umedecidas até $80 \%$ da capacidade de retenção de água e acondicionadas em frascos de vidro com capacidade para $150 \mathrm{~mL}$. Os frascos foram fechados com tampas plásticas perfuradas, para permitir a condição de aerobiose. Posteriormente, os frascos foram colocados, por um período de 90 dias, em uma estufa com temperatura regulada para $27 \pm 1^{\circ} \mathrm{C}$. A cada sete dias, a umidade foi determinada e restabelecida a quantidade inicial de água por meio de uma seringa, introduzindo-se a agulha em vários pontos do solo, depositando-se, assim, a água, sem modificar a acomodação e a estrutura das amostras dentro dos frascos. Cada tratamento foi realizado com três repetições. Neste estudo, por três dias não consecutivos, durante o período de incubação, houve falta de energia elétrica, ficando a incubadora com temperatura em torno de $15^{\circ} \mathrm{C}$. A extração e a quantificação do S-elementar remanescente, não oxidado ao solo, foram realizadas no Departamento de Química Analítica Ambiental do Instituto de Química da Universidade Federal do Rio Grande do Sul. A extração foi realizada agitando-se, por $12 \mathrm{~h}$ em um agitador horizontal, 2,5 g das amostras de solo em solução extratora constituída de $20 \mathrm{~mL}$ de clorofórmio com 99,8 \% de pureza (grau HPLC) mais $10 \mathrm{~mL}$ de água destilada. Após a agitação, foi retirada uma alíquota do extrato (clorofórmio + S-elementar), utilizando-se uma pipeta de Pasteur com lã de vidro em seu interior. Essa alíquota foi diluída 1.000 vezes, com clorofórmio grau HPLC, em balão volumétrico. A concentração de S-elementar na amostra foi determinada comparando-se a leitura cromatográfica com uma curva de resposta obtida com S-elementar (padrão certificado com 99,9\% de pureza) nas concentrações de $1,3,5,7,10$ e $20 \mathrm{mg} \mathrm{L}^{-1}$, com uso de cromatografia líquida de alto desempenho (HPLC). Para determinação do S-elementar na solução extratora, foi utilizado cromatógrafo Shimadzu LC 10AD, nas seguintes condições: detector ultravioleta (UV) a $254 \mathrm{~nm}$; coluna fase reversa PRP-1 (150 x 4,1 mm - Hamilton); temperatura da coluna ambiente: $25 \pm 2{ }^{\circ} \mathrm{C}$; fase móvel clorofórmio:metanol 50:50 (v/v); taxa de fluxo de $1 \mathrm{~mL} \mathrm{~min}^{-1}$; e volume de injeção de $50 \mu \mathrm{L}$. No momento da injeção, as amostras foram filtradas com membranas de $0,45 \mathrm{~mm}$ de diâmetro de poros.
Na determinação da taxa de oxidação foi utilizada a fórmula sugerida por Watkinson (1989) e Ghani et al. (1997): $\mathrm{K}=\left(1-(\mathrm{m} / \mathrm{mo})^{0,33}\right)$. g (bc $)^{0,5 / 2 t}$, em que $\mathrm{K}=$ taxa de oxidação do S-elementar $\left(\mathrm{mg} \mathrm{cm}^{-2} \mathrm{dia}^{-1} \mathrm{de}^{0}\right)$; $\mathrm{m}=$ massa do S-elementar remanescente após o tempo $\mathrm{t} ; \mathrm{mo}=$ massa inicial do S-elementar; $\mathrm{t}=$ tempo de incubação em dias; $\mathrm{g}=$ densidade do $\mathrm{S}\left(=2,07 \mathrm{~g} \mathrm{~cm}^{-3}\right)$; e $(b c)^{0,5}=$ média geométrica do maior e do menor tamanho de partícula de S-elementar.

Para a variável "taxa de oxidação", procedeu-se à análise de variância conforme o modelo: $\mathrm{Y}_{\mathrm{ij}}=\mu+\alpha_{\mathrm{i}}+$ $\varepsilon_{\mathrm{ij}}$ em que, $\mathrm{i}=1,2, \ldots, 42$ (índice de solos) e $\mathrm{j}=1,2,3$ (índice de repetição), sendo $\mathrm{Y}_{\mathrm{ij}}=$ observação da variável avaliada na repetição j do solo $i ; \mu=$ média geral; $\alpha_{i}=$ efeito do solo; e $\varepsilon_{\mathrm{ij}}=$ erro aleatório associado à observação. A verificação de adequação deste modelo de análise de variância foi realizada por meio do teste de homogeneidade de variâncias de Bartlett. A complementação da análise de variância foi feita utilizando-se o teste de Tukey a $5 \%$.

Agrupamento: com o objetivo de formar grupos de solos homogêneos, utilizou-se a técnica multivariada de análise de agrupamento (cluster analysis), considerando-se conjuntamente, para os 42 solos, as variáveis que os caracterizam (variáveis explicativas ou independentes): argila, $\mathrm{pH}, \mathrm{P}, \mathrm{K}$ e S,

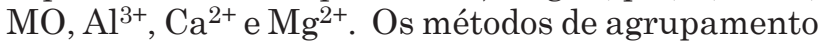
utilizados foram: o de Ward e os do encadeamento simples ("Single Linkage Cluster Analysis") e médio (“Average Linkage Cluster Analysis"). Os três métodos conduziram à formação dos mesmos grupos.

Análise final: para as médias da variável taxa de oxidação, considerando-se os grupos de solos formados por semelhança na análise de agrupamento, procedeu-se à análise de variância, conforme o modelo: $Y_{\mathrm{ij}}=\mu+\alpha_{\mathrm{i}}+\varepsilon_{\mathrm{ij}}$, e a uma análise de covariância, considerando-se os grupos de solos como variável de classificação e as variáveis explicativas ou independentes: argila, pH, P, K e S, MO, $\mathrm{Al}^{3+}, \mathrm{Ca}^{2+}$ e $\mathrm{Mg}^{2+}$ como covariáveis. $\mathrm{O}$ modelo de análise de covariância adotado foi: $Y \mathrm{ij}=\mu+\alpha_{\mathrm{i}}+\sum_{\mathrm{k}=1}^{9} \beta_{\mathrm{k}} \mathrm{X}_{\mathrm{kij}}+\varepsilon_{\mathrm{ij}}$, em que, nos dois modelos, $\mathrm{i}=1,2, \ldots, 4$ ( índice de grupo de solos ) e $\mathrm{j}=1,2, \ldots, 4$ ou 12 ou 14 (índice de solo); sendo $\mathrm{Y}_{\mathrm{ij}}=$ a observação média da variável avaliada no solo j do grupo de solo i ; $\mu=$ média geral; $\alpha_{i}=$ efeito de grupo de solo; $\beta_{\mathrm{k}}(\mathrm{k}=1,2, \ldots, 9)=$ coeficiente de regressão associado às covariáveis argila, $\mathrm{pH}, \mathrm{P}, \mathrm{K}$ e S, MO, $\mathrm{Al}^{3+}, \mathrm{Ca}^{2+}, \mathrm{Mg}^{2+}$, respectivamente; $\mathrm{X}_{\mathrm{ij}}(\mathrm{k}=1,2, \ldots, 9)$ representam as covariáveis argila, $\mathrm{pH}, \mathrm{P}, \mathrm{K}$ e S, MO, $\mathrm{Al}^{3+}, \mathrm{Ca}^{2+} \mathrm{e} \mathrm{Mg}^{2+}$, respectivamente; e $\varepsilon_{\mathrm{ij}}=$ erro aleatório associado à observação.

A adoção do modelo de covariância é justificada, uma vez que a diferença entre grupos de solos, quanto à variável taxa de oxidação, pode ser devida, em parte, à influência das covariáveis argila, pH, P, K e S, MO, $\mathrm{Al}^{3+}, \mathrm{Ca}^{2+} \mathrm{e} \mathrm{Mg}^{2+}$. 
Quadro 1. Atributos das 42 amostras de solos coletadas no horizonte A, na camada de 0-20 cm, no Distrito Federal e em diferentes Estados do Brasil

\begin{tabular}{|c|c|c|c|c|c|c|c|c|c|c|c|}
\hline Número & Solo & Origem & MO & Argila & $\mathrm{pH} \mathrm{H}_{2} \mathrm{O}$ & $\mathbf{P}$ & $\mathbf{K}$ & $\mathrm{S}_{-} \mathrm{SO}_{4}$ & $\mathrm{Al}^{3+}$ & $\mathrm{Ca}^{2+}$ & $\mathrm{Mg}^{2+}$ \\
\hline & & & \multicolumn{2}{|c|}{$\mathrm{g} \mathrm{kg}^{-1}$} & & \multicolumn{2}{|c|}{$-\mathrm{mg} \mathrm{kg}^{-1}$} & $\mathrm{mg} \mathrm{kg}^{-1}$ & \multicolumn{3}{|c|}{$\mathrm{cmol}_{\mathrm{c}} \mathrm{dm}^{-3}-$} \\
\hline 1 & LVA & SP & 30 & 240 & 4,8 & 3,4 & 91 & 13 & 0,8 & 2,2 & 0,9 \\
\hline 2 & LV & $\mathrm{SP}$ & 24 & 360 & 5,2 & 4,1 & 183 & 7,6 & 0,6 & 2,3 & 0,9 \\
\hline 3 & LV & $\mathrm{SP}$ & 25 & 340 & 4,2 & 3,4 & 126 & 7,3 & 2,2 & 13 & 0,6 \\
\hline 4 & PVA & $\mathrm{SP}$ & 11 & 80 & 4,7 & 8,3 & 84 & 8,9 & 0,7 & 1,8 & 0,7 \\
\hline 5 & PVA & SP & 15 & 100 & 6,4 & 52 & 86 & 5,4 & 0 & 3,5 & 1,4 \\
\hline 6 & $\mathrm{TC}$ & $\mathrm{RS}$ & 29 & 80 & 4,7 & 3,1 & 49 & 12 & 1,0 & 2,9 & 1,2 \\
\hline 7 & $\mathrm{TC}$ & $\mathrm{RS}$ & 33 & 130 & 4,4 & 3,4 & 37 & 11 & 1,6 & 3,1 & 1,6 \\
\hline 8 & LV & $\mathrm{RS}$ & 36 & 500 & 5,9 & 9,4 & 211 & 12 & 0 & 7,0 & 3,4 \\
\hline 9 & LV & $\mathrm{RS}$ & 51 & 400 & 6,2 & 4,8 & 114 & 13 & 0 & 19,1 & 6,6 \\
\hline 10 & $\mathrm{SG}$ & $\mathrm{RS}$ & 24 & 80 & 5,3 & 28 & 33 & 6,6 & 0,6 & 4,6 & 2,3 \\
\hline 11 & $\mathrm{SG}$ & $\mathrm{RS}$ & 13 & 120 & 4,7 & 21 & 37 & 6,5 & 1,1 & 2,7 & 1,2 \\
\hline 12 & LVA & $\mathrm{DF}$ & 26 & 280 & 4,3 & 1,6 & 34 & 6,8 & 1,8 & 0,9 & 0,4 \\
\hline 13 & LVA & $\mathrm{DF}$ & 37 & 470 & 4,2 & 1,4 & 28 & 6,2 & 1,3 & 0,7 & 0,3 \\
\hline 14 & $\mathrm{LV}$ & $\mathrm{DF}$ & 41 & 470 & 4,2 & 1,6 & 28 & 6,7 & 1,6 & 0,7 & 0,3 \\
\hline 15 & LV & $\mathrm{RS}$ & 32 & 470 & 4,5 & 2,2 & 192 & 12 & 1,2 & 3,4 & 1,6 \\
\hline 16 & LV & $\mathrm{RS}$ & 32 & 570 & 5,2 & 6,1 & 117 & 9,1 & 0,7 & 5,3 & 2,6 \\
\hline 17 & $\mathrm{LV}$ & $\mathrm{RS}$ & 35 & 560 & 4,5 & 2,7 & 193 & 6,8 & 1,6 & 2,9 & 1,3 \\
\hline 18 & $\mathrm{SG}$ & $\mathrm{RS}$ & 72 & 260 & 5,3 & 20 & 158 & 5,7 & 0,4 & 28,3 & 13,2 \\
\hline 19 & $\mathrm{SG}$ & $\mathrm{RS}$ & 70 & 340 & 5,4 & 16 & 220 & 12 & 0,3 & 28,7 & 12 \\
\hline 20 & LV & $\mathrm{RS}$ & 36 & 470 & 4,9 & 25 & 92 & 14 & 0,8 & 4,1 & 1,7 \\
\hline 21 & $\mathrm{SG}$ & $\mathrm{RS}$ & 43 & 220 & 5,3 & 11 & 108 & 9,9 & 0,5 & 15,3 & 6,6 \\
\hline 22 & PV & $\mathrm{RS}$ & 25 & 220 & 5,3 & 12 & 197 & 7,2 & 0,6 & 3,9 & 1,7 \\
\hline 23 & PV & $\mathrm{RS}$ & 23 & 170 & 4,5 & 2,2 & 78 & 12 & 1,6 & 1,5 & 0,7 \\
\hline 24 & LV & $\mathrm{RS}$ & 15 & 170 & 4,5 & 3,6 & 29 & 9,9 & 1,5 & 0,9 & 0,4 \\
\hline 25 & $\mathrm{LV}$ & $\mathrm{RS}$ & 16 & 150 & 5,4 & 9,7 & 45 & 5,4 & 0,6 & 2,6 & 1,2 \\
\hline 26 & $\mathrm{LV}$ & $\mathrm{DF}$ & 33 & 220 & 6,1 & 21 & 110 & 9,7 & 0 & 5,5 & 2,6 \\
\hline 27 & LVA & $\mathrm{DF}$ & 30 & 340 & 5,0 & 4,2 & 56 & 13 & 0,7 & 3,5 & 1,7 \\
\hline 28 & LV & $\mathrm{RS}$ & 31 & 250 & 4,4 & 3,1 & 85 & 6,3 & 2,1 & 2,2 & 1,0 \\
\hline 29 & LV & $\mathrm{RS}$ & 42 & 400 & 4,9 & 24 & 211 & 13 & 0,7 & 5,2 & 2,6 \\
\hline 30 & LV & $\mathrm{RS}$ & 32 & 280 & 4,0 & 27 & 178 & 16 & 3,4 & 1,3 & 0,7 \\
\hline 31 & LV & $\mathrm{PR}$ & 30 & 540 & 4,7 & 31 & 185 & 5,7 & 1,0 & 4,1 & 1,7 \\
\hline 32 & LV & PR & 42 & 570 & 5,0 & 2,9 & 158 & 8,2 & 0,4 & 5,4 & 2,3 \\
\hline 33 & $\mathrm{LV}$ & $\mathrm{PR}$ & 30 & 80 & 4,9 & 4,1 & 48 & 9,2 & 0,5 & 3,0 & 1,3 \\
\hline 34 & LV & PR & 34 & 130 & 4,8 & 52 & 50 & 7,2 & 0,4 & 3,6 & 1,7 \\
\hline 35 & LV & PR & 38 & 400 & 5,0 & 109 & 63 & 7,8 & 0,5 & 4,6 & 2,1 \\
\hline 36 & LV & $\mathrm{PR}$ & 39 & 510 & 4,7 & 4,7 & 121 & 9,1 & 1,0 & 2,2 & 0,9 \\
\hline 37 & $\mathrm{LV}$ & PR & 44 & 220 & 5,6 & 29 & 182 & 11 & 0,3 & 4,9 & 2,3 \\
\hline 38 & $\mathrm{LV}$ & PR & 43 & 340 & 4,6 & 1,6 & 50 & 7,5 & 1,4 & 1,4 & 0,6 \\
\hline 39 & PVA & $\mathrm{SP}$ & 11 & 70 & 6,9 & 71 & 59 & 6,2 & 0 & 2,8 & 1,2 \\
\hline 40 & LVA & PI & 30 & 260 & 5,2 & 21 & 74 & 12 & 0,5 & 2,0 & 1,0 \\
\hline 41 & LVA & PI & 45 & 480 & 4,6 & 1,7 & 29 & 12 & 2,4 & 0,3 & 0,2 \\
\hline 42 & LVA & PI & 26 & 240 & 5,0 & 1,6 & 15 & 9,7 & 1,2 & 0,2 & 0,3 \\
\hline
\end{tabular}

Legendas: PVA (Argissolo Vermelho-Amarelo); PV (Argissolo Vermelho); LV (Latossolo Vermelho); LVA (Latossolo VermelhoAmarelo); TC (Luvissolo Crômico); SG (Planossolo Hidromórfico).

\section{RESULTADOS E DISCUSSÃO}

A análise estatística mostrou diferenças significativas nos valores das taxas de oxidação do Selementar entre os solos estudados. Estas, após 90 dias de incubação, apresentaram variação de quase 20 vezes para as diferentes amostras. A taxa de oxidação do solo 10 foi a mais baixa, apresentando valor de $1,95 \mu \mathrm{g} \mathrm{cm}^{-2}$ dia $^{-1}$ de $\mathrm{S}^{0}$. O solo 35 foi o que mostrou a maior taxa de oxidação: acima de $21,5 \mu \mathrm{g} \mathrm{cm}^{-2}$ dia $^{-1}$ de $\mathrm{S}^{0}$ (Quadro 2). 
No prosseguimento da análise estatística, agruparam-se os solos com características homogêneas internas (Quadro 3). Esse agrupamento foi feito considerando-se, conjuntamente, para os 42 solos, as vari-

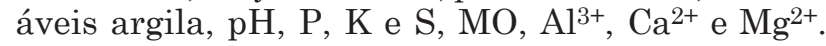

Foram obtidos os seguintes grupos de solos: Grupo 1 solos: 1, 3, 4, 9, 16, 20, 21, 23, 26, 28, 36 e 40; Grupo 2 solos: $6,7,10,11,12,13,14,24,25,27,33,38,41 \mathrm{e}$ 42; Grupo 3 - solos: 5, 34, 35 e 39; e Grupo 4 - solos: 2 , $8,15,17,18,19,22,29,30,31,32$ e 37.

Quadro 2. Taxa de oxidação de S-elementar em amostras de 42 solos coletadas em vários Estados do Brasil, em ordem decrescente, após 90 dias de incubação com esse elemento, à temperatura de $27 \pm 1^{\circ} \mathrm{C}$

\begin{tabular}{|c|c|c|c|c|c|c|c|c|c|c|}
\hline \multirow{2}{*}{$\begin{array}{c}\text { Solo } \\
35\end{array}$} & \multicolumn{10}{|c|}{ Taxa de oxidação $\left(\mu \mathrm{g} \mathrm{cm}^{-2} \mathrm{dia}^{-1}\right.$ de $\left.\mathrm{S}^{0}\right)$} \\
\hline & 21,89 & A & & & & & & & & \\
\hline 32 & 20,43 & $\mathrm{~A}$ & $\mathrm{~B}$ & & & & & & & \\
\hline 26 & 20,39 & $\mathrm{~A}$ & $\mathrm{~B}$ & & & & & & & \\
\hline 18 & 20,12 & A & B & $\mathrm{C}$ & & & & & & \\
\hline 2 & 18,77 & $\mathrm{~A}$ & $\mathrm{~B}$ & $\mathrm{C}$ & $\mathrm{D}$ & & & & & \\
\hline 31 & 18,26 & A & $\mathrm{B}$ & $\mathrm{C}$ & $\mathrm{D}$ & $\mathrm{E}$ & & & & \\
\hline 19 & 17,86 & $\mathrm{~A}$ & $\mathrm{~B}$ & $\mathrm{C}$ & $\mathrm{D}$ & $\mathrm{E}$ & $\mathrm{F}$ & & & \\
\hline 13 & 17,83 & $\mathrm{~A}$ & $\mathrm{~B}$ & $\mathrm{C}$ & $\mathrm{D}$ & $\mathrm{E}$ & $\mathrm{F}$ & & & \\
\hline 34 & 17,70 & $\mathrm{~A}$ & B & $\mathrm{C}$ & $\mathrm{D}$ & $\mathrm{E}$ & $\mathrm{F}$ & & & \\
\hline 8 & 16,32 & $\mathrm{~A}$ & B & $\mathrm{C}$ & $\mathrm{D}$ & $\mathrm{E}$ & $\mathrm{F}$ & $\mathrm{G}$ & & \\
\hline 41 & 16,31 & $\mathrm{~A}$ & B & $\mathrm{C}$ & $\mathrm{D}$ & $\mathrm{E}$ & $\mathrm{F}$ & G & & \\
\hline 29 & 15,27 & $\mathrm{~A}$ & B & $\mathrm{C}$ & $\mathrm{D}$ & $\mathrm{E}$ & $\mathrm{F}$ & $\mathrm{G}$ & $\mathrm{H}$ & \\
\hline 15 & 14,14 & $\mathrm{~A}$ & B & $\mathrm{C}$ & $\mathrm{D}$ & $\mathrm{E}$ & $\mathrm{F}$ & $\mathrm{G}$ & $\mathrm{H}$ & I \\
\hline 38 & 13,83 & $\mathrm{~A}$ & B & $\mathrm{C}$ & $\mathrm{D}$ & $\mathrm{E}$ & $\mathrm{F}$ & G & $\mathrm{H}$ & I \\
\hline 36 & 13,52 & $\mathrm{~A}$ & B & $\mathrm{C}$ & $\mathrm{D}$ & $\mathrm{E}$ & $\mathrm{F}$ & G & $\mathrm{H}$ & I \\
\hline 9 & 13,25 & $\mathrm{~A}$ & B & $\mathrm{C}$ & $\mathrm{D}$ & $\mathrm{E}$ & $\mathrm{F}$ & $\mathrm{G}$ & $\mathrm{H}$ & I \\
\hline 1 & 13,02 & $\mathrm{~A}$ & B & $\mathrm{C}$ & $\mathrm{D}$ & $\mathrm{E}$ & $\mathrm{F}$ & $\mathrm{G}$ & $\mathrm{H}$ & I \\
\hline 17 & 12,96 & $\mathrm{~A}$ & B & $\mathrm{C}$ & $\mathrm{D}$ & $\mathrm{E}$ & $\mathrm{F}$ & G & $\mathrm{H}$ & I \\
\hline 16 & 12,87 & A & B & $\mathrm{C}$ & $\mathrm{D}$ & $\mathrm{E}$ & $\mathrm{F}$ & G & $\mathrm{H}$ & I \\
\hline 20 & 11,83 & $\mathrm{~A}$ & B & $\mathrm{C}$ & $\mathrm{D}$ & $\mathrm{E}$ & $\mathrm{F}$ & $\mathrm{G}$ & $\mathrm{H}$ & I \\
\hline 3 & 11,33 & A & B & $\mathrm{C}$ & $\mathrm{D}$ & $\mathrm{E}$ & $\mathrm{F}$ & $\mathrm{G}$ & $\mathrm{H}$ & I \\
\hline 21 & 11,26 & $\mathrm{~A}$ & B & $\mathrm{C}$ & $\mathrm{D}$ & $\mathrm{E}$ & $\mathrm{F}$ & $\mathrm{G}$ & $\mathrm{H}$ & I \\
\hline 22 & 9,29 & A & B & $\mathrm{C}$ & $\mathrm{D}$ & $\mathrm{E}$ & $\mathrm{F}$ & $\mathrm{G}$ & $\mathrm{H}$ & I \\
\hline 41 & 8,62 & & B & $\mathrm{C}$ & $\mathrm{D}$ & $\mathrm{E}$ & $\mathrm{F}$ & $\mathrm{G}$ & $\mathrm{H}$ & I \\
\hline 12 & 8,08 & & B & $\mathrm{C}$ & $\mathrm{D}$ & $\mathrm{E}$ & $\mathrm{F}$ & $\mathrm{G}$ & $\mathrm{H}$ & I \\
\hline 14 & 8,01 & & B & $\mathrm{C}$ & $\mathrm{D}$ & $\mathrm{E}$ & $\mathrm{F}$ & G & $\mathrm{H}$ & I \\
\hline 25 & 7,80 & & B & $\mathrm{C}$ & $\mathrm{D}$ & $\mathrm{E}$ & $\mathrm{F}$ & G & $\mathrm{H}$ & I \\
\hline 24 & 7,20 & & & $\mathrm{C}$ & $\mathrm{D}$ & $\mathrm{E}$ & $\mathrm{F}$ & G & $\mathrm{H}$ & I \\
\hline 33 & 7,04 & & & & $\mathrm{D}$ & $\mathrm{E}$ & $\mathrm{F}$ & $\mathrm{G}$ & $\mathrm{H}$ & I \\
\hline 28 & 6,69 & & & & $\mathrm{D}$ & $\mathrm{E}$ & $\mathrm{F}$ & $\mathrm{G}$ & $\mathrm{H}$ & I \\
\hline 39 & 6,66 & & & & $\mathrm{D}$ & $\mathrm{E}$ & $\mathrm{F}$ & $\mathrm{G}$ & $\mathrm{H}$ & I \\
\hline 6 & 6,51 & & & & $\mathrm{D}$ & $\mathrm{E}$ & $\mathrm{F}$ & $\mathrm{G}$ & $\mathrm{H}$ & I \\
\hline 5 & 5,55 & & & & & $\mathrm{E}$ & $\mathrm{F}$ & G & $\mathrm{H}$ & I \\
\hline 4 & 5,08 & & & & & & $\mathrm{~F}$ & $\mathrm{G}$ & $\mathrm{H}$ & I \\
\hline 7 & 4,92 & & & & & & $\mathrm{~F}$ & G & $\mathrm{H}$ & I \\
\hline 23 & 4,26 & & & & & & & $\mathrm{G}$ & $\mathrm{H}$ & I \\
\hline 11 & 3,90 & & & & & & & $\mathrm{G}$ & $\mathrm{H}$ & I \\
\hline 30 & 3,74 & & & & & & & G & $\mathrm{H}$ & I \\
\hline 42 & 3,37 & & & & & & & $\mathrm{G}$ & $\mathrm{H}$ & I \\
\hline 40 & 2,52 & & & & & & & & $\mathrm{H}$ & I \\
\hline 27 & 2,40 & & & & & & & & $\mathrm{H}$ & I \\
\hline 10 & 1,95 & & & & & & & & & I \\
\hline
\end{tabular}

Coeficiente de variação $=35,71 \%$. Médias seguidas pela mesma letra não diferem entre si (Tukey, $5 \%$ ). 
Quadro 3. Grupos de solos e suas características homogêneas internas

\begin{tabular}{|c|c|c|c|c|c|}
\hline Grupo & Número de observações & Variável $^{(1)}$ & Média & Mínimo & Máximo \\
\hline \multirow[t]{9}{*}{ Grupo 1} & 12 & Argila & 310 & 80 & 560 \\
\hline & & $\mathrm{pH}$ & 5,02 & 4,20 & 6,20 \\
\hline & & $\mathrm{P}$ & 9,50 & 2,20 & 25,00 \\
\hline & & $\mathrm{K}$ & 100,00 & 74,00 & 126,00 \\
\hline & & $\mathrm{s}$ & 10,36 & 6,30 & 14,00 \\
\hline & & MO & 32 & 11 & 51 \\
\hline & & $\mathrm{Al}^{3+}$ & 0,91 & 0,00 & 2,20 \\
\hline & & $\mathrm{Ca}^{2+}$ & 6,18 & 1,50 & 19,10 \\
\hline & & $\mathrm{Mg}^{2+}$ & 2,16 & 0,60 & 6,60 \\
\hline \multirow[t]{9}{*}{ Grupo 2} & 14 & Argila & 245 & 80 & 480 \\
\hline & & $\mathrm{pH}$ & 4,70 & 4,20 & 5,40 \\
\hline & & $\mathrm{P}$ & 6,19 & 1,40 & 28,00 \\
\hline & & $\mathrm{K}$ & 37,00 & 15,00 & 56,00 \\
\hline & & $\mathrm{s}$ & 8,74 & 5,40 & 13,00 \\
\hline & & MO & 29 & 13 & 45 \\
\hline & & $\mathrm{Al}^{3+}$ & 1,24 & 0,50 & 2,40 \\
\hline & & $\mathrm{Ca}^{2+}$ & 1,96 & 0,20 & 4,60 \\
\hline & & $\mathrm{Mg}^{2+}$ & 0,93 & 0,20 & 2,30 \\
\hline \multirow[t]{9}{*}{ Grupo 3} & 4 & Argila & 175 & 70 & 400 \\
\hline & & $\mathrm{pH}$ & 5,78 & 4,80 & 6,90 \\
\hline & & $\mathrm{P}$ & 71,00 & 52,00 & 109,00 \\
\hline & & $\mathrm{K}$ & 64,50 & 50,00 & 86,0 \\
\hline & & $\mathrm{s}$ & 6,65 & 5,40 & 7,80 \\
\hline & & MO & 25 & 11 & 38 \\
\hline & & $\mathrm{Al}^{3+}$ & 0,23 & 0,00 & 0,50 \\
\hline & & $\mathrm{Ca}^{2+}$ & 3,63 & 2,80 & 4,60 \\
\hline & & $\mathrm{Mg}^{2+}$ & 1,60 & 1,20 & 2,10 \\
\hline \multirow[t]{9}{*}{ Grupo 4} & 12 & Argila & 393 & 220 & 570 \\
\hline & & $\mathrm{pH}$ & 5,03 & 4,00 & 5,90 \\
\hline & & $\mathrm{P}$ & 15,03 & 2,20 & 31,00 \\
\hline & & $\mathrm{K}$ & 189,00 & 158,00 & 220,00 \\
\hline & & $\mathrm{S}$ & 9,77 & 5,70 & 16,0 \\
\hline & & MO & 40 & 24 & 72 \\
\hline & & $\mathrm{Al}^{3+}$ & 0,88 & 0,00 & 3,40 \\
\hline & & $\mathrm{Ca}^{2+}$ & 8,12 & 1,30 & 28,70 \\
\hline & & $\mathrm{Mg}^{2+}$ & 3,64 & 0,70 & 13,20 \\
\hline
\end{tabular}

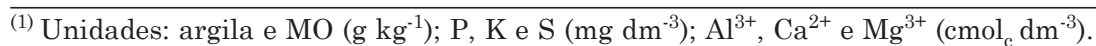

Grupo 1 - solos: 1, 3, 4, 9, 16, 20, 21, 23, 26, 28, 36 e 40; Grupo 2 - solos: 6, 7, 10, 11, 12, 13, 14, 24, 25, 27, 33, 38, 41 e 42; Grupo 3 solos: 5, 34, 35 e 39; e Grupo 4 - solos: $2,8,15,17,18,19,22,29,30,31,32$ e 37.

Caracterizando cada um dos grupos de solos quanto às suas variáveis explicativas ou independentes, argila, $\mathrm{pH}, \mathrm{P}, \mathrm{K}$ e S, $\mathrm{MO}, \mathrm{Al}^{3+}, \mathrm{Ca}^{2+}, \mathrm{Mg}^{2+}$, foram obtidos os valores para a média, para o mínimo e para o máximo.

Os quatro grupos de solo caracterizaram-se por apresentar teores baixo, médio e alto para as variáveis explicativas ou independentes, conforme observado no quadro 4. Para estes grupos, obtiveram-se, para a taxa de oxidação, diferenças significativas, com o grupo 4 se destacando como o que apresentou solos com maiores taxas de oxidação, e o grupo 2 , com valores inferiores (Quadro 5).

Com o objetivo de verificar se as diferenças das taxas de oxidação entre solos e grupos foram devidas aos atributos físicos e químicos dos solos, foi realizada uma análise estatística de covariância. Os resultados mostraram que a diferença, pelo menos em parte, se deveu ao efeito das covariáveis argila, $\mathrm{pH}, \mathrm{P}, \mathrm{K} \mathrm{e} \mathrm{S}$, $\mathrm{MO}, \mathrm{Al}^{3+}, \mathrm{Ca}^{2+}, \mathrm{Mg}^{2+}$. Isso ocorre porque, eliminando- 
se o efeito das covariáveis, não se verificaram diferenças entre os grupos $(P=0,8812)$. Entre as covariáveis, eliminando-se o efeito de grupo de solo, o $\mathrm{Al}^{3+}(\mathrm{P}=0,0048)$, a $\mathrm{MO}(\mathrm{P}=0,0105)$ e o $\mathrm{S}(\mathrm{P}=0,0538)$ são significativamente importantes para explicar a variação dos resultados. As estimativas dos coeficientes que relacionam a taxa de oxidação com as covariáveis são apresentadas no quadro 6.
Os resultados obtidos mostraram diferenças acentuadas entre os 42 solos para a capacidade de oxidar S-elementar. A análise estatística mostrou que o fator que se correlacionou de forma significativa positiva com a taxa de oxidação do S-elementar dos 42 solos foi a MO, ao passo que os fatores que se correlacionaram negativamente foram $\mathrm{Al}^{3+} \mathrm{e} \mathrm{S}$ do solo. Os coeficientes de determinação foram de 0,095 \% para

Quadro 4. Classificação de cada grupo de acordo com suas características

\begin{tabular}{|c|c|c|c|c|}
\hline Variáveis & Grupo 1 & Grupo 2 & Grupo 3 & Grupo 4 \\
\hline Argila & Médio & Médio & Baixo & Alto \\
\hline $\mathrm{pH}$ & Médio & Médio & Alto & Médio \\
\hline $\mathrm{P}$ & Baixo & Baixo & Alto & Médio \\
\hline $\mathrm{K}$ & Médio & Baixo & Médio & Alto \\
\hline $\mathrm{S}$ & Médio & Médio & Baixo & Médio \\
\hline MO & Médio & Médio & Baixo & Alto \\
\hline $\mathrm{Al}^{3+}$ & Médio & Médio & Baixo & Médio \\
\hline $\mathrm{Ca}^{2+}$ & Alto & Baixo & Médio & Alto \\
\hline $\mathrm{Mg}^{2+}$ & Médio & Médio & Médio & Alto \\
\hline
\end{tabular}

Quadro 5. Valor de taxa de oxidação do S-elementar obtido para grupos de solos com características internas diferentes

Grupo Taxa de oxidação $\left(\mu \mathrm{g} \mathrm{cm}^{-2} \operatorname{dia}^{-1} \operatorname{de~} \mathrm{S}^{0}\right)$

\begin{tabular}{ll}
\hline & $15,29 \mathrm{~A}$ \\
3 & $12,96 \mathrm{AB}$ \\
1 & $10,51 \mathrm{AB}$ \\
2 & $7,25 \mathrm{~B}$ \\
\hline
\end{tabular}

Médias seguidas pela mesma letra não diferem entre si (Tukey, $5 \%$ ).

Quadro 6. Coeficientes de determinação obtidos para a taxa de oxidação de S-elementar e atributos selecionados para os 42 solos

\begin{tabular}{lccc}
\hline Grupo e atributos ${ }^{(1)}$ & $\mathbf{R}$ & $\mathbf{R}^{2}$ & $\mathbf{P}$ \\
\hline Grupo & 0,09 & 0,008 & 0,8812 \\
Argila & 0,15 & 0,025 & 0,1679 \\
$\mathrm{pH}$ & $-0,18$ & 0,032 & 0,1206 \\
$\mathrm{P}$ & 0,09 & 0,008 & 0,4830 \\
$\mathrm{~K}$ & 0,03 & 0,0006 & 0,8322 \\
$\mathrm{~S}^{-S O} \mathrm{M}_{4}$ & $-0,22$ & 0,052 & 0,0538 \\
$\mathrm{MO}^{3+}$ & 0,30 & 0,095 & 0,0105 \\
$\mathrm{Ca}^{2+}$ & $-0,35$ & 0,120 & 0,0048 \\
$\mathrm{Mg}^{2+}$ & 0,09 & 0,008 & 0,4101 \\
\end{tabular}

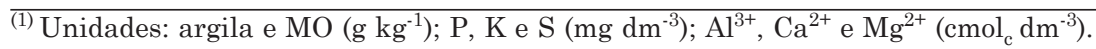


a MO, 0,120 para o $\mathrm{Al}^{3+}$ e 0,052 para o S. A ausência de alta correlação específica indica que os atributos isoladamente não foram importantes e sim, provavelmente, a interação entre vários atributos do solo. Esses resultados concordam com os de Janzen \& Bettany (1987a), que obtiveram, para 39 solos do Canadá, baixas correlações entre as características dos solos e suas taxas de oxidação. No quadro 4 destaca-se o grupo 4, que apresentou as maiores taxas de oxidação e foi o único em que não havia nenhum dos seus atributos classificados como "baixo". Isso demonstra que, provavelmente, a interação entre os fatores é mais importante para a taxa de oxidação do S-elementar do que um atributo isolado.

$\mathrm{O}$ teor de MO do solo afetou a taxa de oxidação, resultado que concorda com o verificado por Janzen \& Bettany (1987a) em estudo com 39 solos, os quais constataram significância positiva entre a oxidação do S-elementar e o teor de MO do solo. Lawrence \& Germida (1988) também observaram efeito benéfico da MO no solo na oxidação do S-elementar. A relação positiva entre a oxidação do S-elementar e a MO pode ser atribuída à resposta de organismos heterotróficos que oxidam o S-elementar, utilizando o substrato disponível como fonte de energia (Wainwright et al., 1986; Lawrence \& Germida 1988; Cifuentes \& Lindemann, 1993; Cowell \& Schoenau, 1995). Portanto, é provável que, pelo menos em parte, a oxidação de S-elementar em solos do Brasil seja feita por microrganismos heterotróficos, dependentes de MO para realizar a oxidação.

Não foram encontradas na literatura informações sobre a relação do $\mathrm{Al}^{3+}$ e a taxa de oxidação do $\mathrm{S}$ elementar. Essa relação pode ser indicativo de que a concentração de $\mathrm{H}^{+}$não afeta, diretamente, a taxa de oxidação do S. Existe relação inversa entre o pH e o $\mathrm{Al}$ trocável no solo. Com o aumento do $\mathrm{pH}, \mathrm{o} \mathrm{Al}$ trocável diminui. Assim, é possível que a taxa de oxidação seja afetada pelo $\mathrm{Al}$ trocável e não pelo $\mathrm{pH}$ do solo. Logo, a adição de corretivos de acidez aos solos aumenta o pH, o que, indiretamente, reduz o $\mathrm{Al}$ trocável, aumentando a taxa de oxidação.

Outro fator que afetou negativamente a taxa de oxidação do S-elementar foi o teor de S do solo. Esse resultado contradiz o obtido por Janzen \& Bettany (1987a), que não encontraram correlação significativa entre o teor inicial de $\mathrm{S}$ do solo e a taxa de oxidação para 39 solos do Canadá.

O teor de argila do solo não afetou a taxa de oxidação do S nos 42 solos. Segundo Germida \& Janzen (1993), a oxidação do S-elementar é afetada por propriedades físicas do solo, apesar de esses efeitos nem sempre serem consistentes. Os resultados obtidos neste estudo contrariam algumas investigações, nas quais se concluiu que as taxas de oxidação foram inversamente relacionadas ao teor de argila e diretamente relacionadas ao teor de areia (Janzen \& Bettany, 1987a; Lawrence \& Germida, 1988; Deng \& Dick, 1990). Entretanto, concordam com outros trabalhos que não obtiveram relações significativas entre a textura do solo e a taxa de oxidação de S-elementar (Rehm \& Cadwell, 1969; McCaskill \& Blair, 1987; Watkinson, 1989). Segundo Germida \& Janzen (1993), essas observações aparentemente contraditórias são, provavelmente, reflexos indiretos do estado de aeração dos solos, e o efeito inconsistente da textura pode ser parcialmente explicado pelas diferentes condições de umidade em vários experimentos.

$\mathrm{O} \mathrm{pH}$ também foi um fator que não afetou a taxa de oxidação do S-elementar, o que contradiz as afirmações de Janzen \& Bettany (1987b), de Lawrence \& Germida (1988) e de Nor \& Tabatabai (1977), segundo as quais o pH do solo é, quase sempre, relacionado positivamente com a taxa de oxidação do S-elementar. Isso porque o efeito positivo do alto $\mathrm{pH}$ na oxidação do S-elementar deve estar relacionado à capacidade do solo em tamponar o ácido sulfúrico formado na oxidação, que, se acumulado em altas concentrações, inibe a atividade dos microrganismos que transformam o S-elementar em S-sulfato (Fox et al., 1964; Barrow, 1971). É provável que o efeito positivo dos valores mais elevados de $\mathrm{pH}$ na oxidação do $\mathrm{S}$ elementar seja devido à redução do $\mathrm{Al}$ trocável e não à redução da concentração de $\mathrm{H}^{+}$na solução do solo.

Os resultados deste estudo também demonstraram que o teor de P não afetou a oxidação, confirmando os resultados obtidos em outro estudo não publicado. A falta de significância estatística entre a taxa de oxidação e os teores de $\mathrm{P}$ contraria vários outros trabalhos que mostraram efeito consistente entre esses fatores (Janzen \& Bettany, 1987a; Lawrence \& Germida, 1988; Lefroy et al., 1997; Sholeh et al., 1997). Contudo, outros autores relatam não haver tal correlação (Lee et al., 1987), referendando os dados aqui obtidos.

Outros nutrientes $\left(\mathrm{Ca}^{2+}, \mathrm{Mg}^{2+}\right.$ e $\left.\mathrm{K}\right)$ foram investigados neste estudo. Nenhum deles teve correlação com a taxa de oxidação, confirmando os resultados de Watkinson (1989), de que a deficiência de nutrientes é raramente um fator que determina os valores das taxas de oxidação do S-elementar.

A ausência de uma relação bem definida e a observação de resultados contraditórios entre a oxidação do S-elementar e as várias propriedades físicas e químicas do solo não implicam, necessariamente, que esses efeitos não sejam importantes. É provável que a oxidação seja governada pela interação desses fatores e não apenas por um fator isolado, exceto em casos extremos (Germida \& Janzen, 1993).

\section{CONCLUSÕES}

1. A capacidade de oxidação de S-elementar foi verificada para todos os solos. As taxas de oxidação obtidas variaram, para as 42 amostras de solos, entre 1,95 e $21,89 \mu \mathrm{g} \mathrm{cm}^{-2} \mathrm{dia}^{-1}$ de $\mathrm{S}^{0}$. 
2. A taxa de oxidação do S-elementar se correlacionou positivamente com o teor de $\mathrm{MO}$ e negativamente com os teores de $\mathrm{Al}$ trocável e de $\mathrm{S}_{-} \mathrm{SO}_{4}$ dos solos.

3. $\mathrm{O} \mathrm{pH}$ e os teores de argila, $\mathrm{P}, \mathrm{K}, \mathrm{Ca}^{2+}{\mathrm{e} \mathrm{Mg}^{2+}}^{2+}$ não afetaram a taxa de oxidação do S-elementar nos solos.

\section{LITERATURA CITADA}

BARROW, N.J. Slowly available sulphur fertilizers in southwestern Australia. I.Elemental sulphur. Aust. J. Exp. Agric. An. Husb., 2:211-216, 1971.

CIFUENTES, F.R. \& LINDEMANN, W.C. Organic matter stimulation of elemental sulfur oxidation in a calcareous soil. Soil Sci. Soc. Am. J., 57:727-731, 1993.

COWELL, L.E. \& SCHOENAU, J.J. Stimulation of elemental sulphur oxidation by sewage sludge. Can. J. Soil Sci., 75:247-249, 1995.

DENG, S. \& DICK, R.P. Sulfur oxidation and rhodanese activity in soils. Soil Sci., 150: 552-560, 1990.

EDMEADES, D.C.; SINCLAIR, A.G.; WATKINSON, J.H.; LEDGARD, S.F.; GHANI, A.; THORROLD, B.S.; BOSWELL, C.C.; BRAITHWAITE, A.C. \& BROWN, M.W Some recent developments in sulphur research in New Zealand agriculture. Sulphur Agric., 18:3-8, 1994.

FOX, R.L.; ATESALP, H.M.; KAMPBELL, D.H. \& RHOADES, H.F. Factors influencing the availability of sulfur fertilizers to alfafa and corn. Madison, Soil Science Society Proceedings, 1964. p.406-408.

GERMIDA, J.J. \& JANZEN, H.H. Factors affecting the oxidation of elemental sulfur in soils. Fert. Res.,35:101114, 1993.

GHANI, A.; WATKINSON, J.H. \& UPSDELL, M.P. Modeling the oxidation of elemental $\mathrm{S}$ in New Zealand pastoral soils. Sulphur Agric., 20:3-9, 1997.

GRAYSTON, S.J. \& GERMIDA, J.J. Influence of crop rhizospheres on populations and activity of heterotrophic sulfur-oxidizing microorganisms. Soil Biol. Biochem., 22:457-463, 1990.

JANZEN, H.H. \& BETTANY, J.R. Measurement of sulfur oxidation in soils. Soil Sci., 143:444-452, 1987a.
JANZEN, H.H. \& BETTANY, J.R. Oxidation of elemental sulfur under field conditions in Central Saskatchewan. Can. J. Soil Sci., 67:609-618, 1987b.

KELLER, P. The effect of sodium chloride and sulphate on sulphur oxidation in soil. Plant Soil, 30:15-22, 1969.

LAWRENCE, J.R. \& GERMIDA, J.J. Relationship between microbial biomass and elemental sulfur oxidation in agricultural soils. Soil Sci. Soc. Am. J., 52:672-677, 1988.

LEE, A.; WATKINSON, J.H.; ORBELL, G.; BAGYARAJ, J. \& LAUREN, D.R. Factors influencing dissolution of phosphate rock and oxidation of elemental sulphur in some New Zealand soils. New Zealand J. Agric. Res., 30:373-385, 1987.

LEFROY, B.; SHOLEH, R.D. \& BLAIR, G.J. Influence of sulfur and phosphorus placement, and sulfur particle size, on elemental sulfur oxidation and the growth response of maize (Zea mays). Austr. J. Agric. Res., 48:485-495, 1997.

MCCASKILL, M.R. \& BLAIR, G.J. Particle size and soil texture effects on elemental sulfur oxidation. Agron.J., 79:1079$1083,1987$.

NOR, Y.M. \& TABATABAI, M.A. Oxidation of elemental sulfur in soils. Soil Sci. Soc. Am. J., 41:736-741, 1977.

REHM, G.W. \& CALDWELL, A.C. Sulfur supplying capacity of soils and the relationship to soil type. Soil Sci., 105:355$361,1968$.

SHOLEH, R.D.; LEFROY, B. \& BLAIR, G.J. Effect of nutrients and elemental sulfur particle size on elemental sulfur oxidation and the growth of Thiobacillus thiooxidans. Austr. J. Agric. Res., 48:497-501, 1997.

TEDESCO, M.J.; GIANELLO, C.;BISSANI, C.A.; BOHNEN, H. \& VOLKWEISS, S.J. Análises de solo, plantas e outros materiais. 2 ed. Porto Alegre, Universidade Federal do Rio Grande do Sul, 1995. 174p. (Boletim Técnico de Solos)

WAINWRIGHT, M.; NEVELL, W. \& GRAYSTON, S.J. Effects of organic matter on sulphur oxidation in soil and influence of sulphur oxidation on soil nitrification. Plant Soil, 96:369376,1986 .

WATKINSON, J.H. Measurement of the oxidation rate of elemental sulfur in soil. Austr. J. Soil Res., 27:365-375, 1989.

ZHAO, F.J.; LOKE, S.Y.; CROSLAND, A.R. \& MCGRATH, S.P. Method to determine elemental sulphur in soils applied to measure sulphur oxidation. Soil Biol. Biochem., 28:1083-1087, 1996. 